\title{
Which One is More Effective, Filgrastim or Lenograstim, During Febrile Neutropenia Attack in Hospitalized Patients with Solid Tumors?
}

\author{
Ozlem Uysal Sonmez ${ }^{1 *}$, Ertugrul Guclu², Ummugul Uyeturk ${ }^{4}$, Onur Esbah ${ }^{3}$, \\ Ibrahim Türker ${ }^{3}$, Oznur Bal $^{3}$, Burcin Budakoglu ${ }^{3}$, Ulku Yalcintas Arslan ${ }^{3}$, Oguz \\ Karabay $^{2}$, Berna Oksuzoglu ${ }^{3}$
}

\begin{abstract}
Background: Chemotherapy-induced febrile neutropenia (FN) with solid tumors causes mortality and morbidity at a significant rate. The purpose of this study was to compare the effects of filgastrim and lenograstim started with the first dose of antibiotics in hospitalized patients diagnosed with FN. Materials and Methods: Between February 2009 and May 2012, 151 patients diagnosed with FN were evaluated, retrospectively. In those considered appropriate for hospitalization, convenient antibiotic therapy with granulocyte colony stimulating factors was started within first 30 minutes by completing necessary examinations in accordance with FEN guide recommendations. Results: In this study, 175 febrile neutropenia attacks in 151 patients were examined. Seventy three of the patients were male and 78 were female. The average age was 53.6 and 53.6, respectively. The most common solid tumor was breast carcinoma in $38(25 \%)$. One hundred and five $\mathrm{FN}$ patients $(\mathbf{5 8 \%})$ were those who received granulocyte colony stimulating factors as primary prophylaxis. Conclusions: While studies comparing both drugs generally involve treatments started for prophylaxis, this study compared the treatment given during the febrile neutropenia attack. Compared to lenograstim, filgastrim shortens the duration of hospitalization during febrile neutropenia attack by facilitating faster recovery with solid tumors.
\end{abstract}

Keywords: Filgrastim - lenograstim - febrile neutropenia - solid tumor - recovery

Asian Pac J Cancer Prev, 16 (3), 1185-1189

\section{Introduction}

The chemotherapy-induced neutropenia causes mortality and morbidity at a significant rate (Aapro et al., 2011; Fontanella et al., 2014). Fever is frequently observed during chemotherapy-induced neutropenia. Neutropenia-related fever could be encountered for at least once in $10-50 \%$ of patients with solid tumor and $>80 \%$ of patients with hematological malignancy (Freifeld et al., 2011). In patients with febrile neutropenia (FN), it is generally required to decrease the dose of chemotherapy drugs or delay the treatment, which causes the failure of implementing an efficient treatment (Aapro et al., 2011; Freifeld et al., 2011).

In the primary prophylaxis, granulocyte-colony stimulating factors (G-CSFs) are started with the first dose of chemotherapy before the presence of neutropenia and fever. Primary prophylaxis decreases the risk of chemotherapy-induced febrile neutropenia in patients with solid tumor or lymphoma (Cooper et al., 2011). However, since this approach brings along a high additional cost, it should be initiated in selected special patient groups. Primary prophylaxis is suggested for patients with more than $20 \%$ possibility of developing febrile neutropenia. Prophylaxis could also be started in individuals with additional risk factors such as old patients or those who have previously had a fever or neutropenia attack, those with a weak performance or nutrition, those receiving no antibiotic prophylaxis, those with a comorbid disease and patients with the risk of fever and/or severe infection during neutropenia. Prophylaxis is not suggested for patients with a febrile neutropenia risk of less than $10 \%$ (Aapro et al., 2011; Freifeld et al., 2011). Filgrastim includes G-CSFs that have the usage approval of lenograstim and pegfilgrastim. It was reported that cases that were given lenograstim and prophylaxis had less febrile neutropenia attacks compared to those that were given filgrastim (Orciuolo et al., 2011).

However, guides state the possibility of using any G-CSFs in order to prevent febrile neutropenia and febrile neutropenia-related complications in patients with indication. 
It was reported that the administration of G-CSF along with the antibiotic treatment during attack of chemotherapy-induced febrile neutropenia decreased both the hospitalization and antibiotherapy duration and accelerated the neutropenia recovery (Clark et al., 2005; Osmani et al., 2012). However, these results were emphasized to be dissatisfactory (Freifeld et al., 2011).

The purpose of this study was to evaluate the general characteristics of patients followed up with the diagnosis of febrile neutropenia and compare the efficiencies of filgrastim and lenograstim that were started with the first dose of antibiotics during the febrile neutropenia attack.

\section{Materials and Methods}

This study was conducted in medical oncology services of Dr. Abdurahman Yurtarslan Ankara Oncology Hospital and Abant Izzet Baysal University Medical Faculty, and Infectious Diseases clinics of Sakarya University Training and Research Hospital. All patients who were older than 18 and were hospitalized with the diagnosis of febrile neutropenia while receiving chemotherapy(ct) were included in the study between February 2009 and May 2012. Patient information was retrospectively obtained from the patient files.

A case form was prepared, which involved the following; demographic data of patients (age, gender, primary diagnosis, chemotherapy drugs received, initial dose of chemotherapy drugs, state of bone marrow involvement, comorbid diseases, whether primary or secondary G-CSF prophylaxis was taken or not), laboratory findings during the hospitalization (leukocyte, neutrophil, platelet), information about the diagnosis and treatment of febrile neutropenia (on which chemotherapy cycle the neutropenia was observed, on which day following the chemotherapy cycle the FEN was diagnosed, the rank of hospitalization with diagnosis of the FEN, days of treatment and recovery from fever and neutropenia, antibiotics that were started for treatment and their intake durations, which G-CSF was given with antibiotherapy and for how long it was given, whether a microbiological diagnosis was established or not, whether a blood and/or blood product transfusion was made or not). This form was sent to the centers, which were involved in the study, by asking them to fill out it. The completed forms were sent to the coordinator center via an electronic mail.

Patients receiving primary or secondary G-CSF prophylaxis were evaluated as the group receiving prophylaxis. Primary G-CSF prophylaxis was given to patients older than 65 receiving the chemotherapy with a high bone marrow toxicity. Secondary G-CSF prophylaxis was accepted as G-CSF, which was started for patients who had developed neutropenic fever during their previous chemotherapy, within 24-48 hours following the chemotherapy in order to prevent the development of neutropenia in following chemotherapies (Keskin et al., 2012).

The diagnosis of febrile neutropenia was accepted as the fact that patients with an absolute neutrophil count of $500 / \mathrm{mm} 3$ or below had a single body temperature measurement of $\geq 38.3^{\circ} \mathrm{C}$ when taken orally or a constant temperature of $\geq 38.0^{\circ} \mathrm{C}$ for 1 hour (Freifeld et al., 2011). A blood culture was received from both peripheral veins of each patient hospitalized in the service with the diagnosis of febrile neutropenia and their complete urinary analysis and posteroanterior chest radiography were performed. Additionally, microbiological examinations were performed on the required areas according to the complaints and physical examination findings of the case. Just after receiving the samples within the first 30 minutes following the hospitalization for the required examinations, antibiotherapy was started in line with the FEN guide recommendations (Hughes et al., 2002) $5 \mathrm{mcg} /$ $\mathrm{kg}$ /day G-CSF was started for almost all of the patients simultaneously with the antibiotics without considering the MASCC score and it was used until the absolute neutrophil count exceeded $5000 / \mathrm{mm}^{3}$.

\section{Statistical methods}

While frequency tables were presented for categorical variables, descriptive statistics (mean, standard deviation, median, minimum, maximum) were presented for digital variables. Cross-table statistics were presented for intergroup categorical comparisons, and their significance levels were determined by using the chi-square test and Fisher's exact test. Regarding digital data that were not normally distributed, Mann Whitney U test was used for independent group comparisons. The statistical significance level was accepted as $\mathrm{p}<0.05$. SPSS 15.0 for Windows was used to conduct statistical analysis.

\section{Results}

This study examined 180 febrile neutropenia attacks observed in 151 patients. 73 of the patients were male and 78 were female. While male patients had an age average of 53.82, female cases had an age average of 53.66. The most frequent malignancy was the breast carcinoma (38 patients, $25.2 \%$ ). While 36 of the breast carcinoma cases were female, 2 were male. The most frequent malignancy in men was lung (17 cases, $23.3 \%$ ) and gastric carcinoma (12 cases, 16.4\%). Bone marrow involvement was investigated on 76 cases and determined in 8 cases. While 25 cases started to receive a decreased dose of chemotherapy due to various reasons, 126 cases received an exact-dose chemotherapy.

While $105(58.3 \%)$ of febrile neutropenia attacks were determined in cases that started to receive prophylactic G-CSF, 75 (42.7\%) were determined in cases that did not receive prophylaxis. While $73(40.6 \%)$ of attacks were observed following the first chemotherapy cycle, 133 (73.9\%) were observed during the first 3 chemotherapy cycles. Febrile neutropenia attacks started averagely 9.91 days after the chemotherapy cycle. On the day when diagnosis of the febrile neutropenia was established; while 132 cases had a leukocyte value of $<1000,57$ cases had a platelet value of $<75.000$. The mean number of days with fever was 2.36 during attacks, the duration of recovery from neutropenia was 3.51 days and the duration of hospitalization was 7.74 days. Pathogenic bacteria were determined in cultures received in 24 cases and the mean duration of using antibiotics was 7.46 days. Blood and 
Which One is More Effective Filgrastim or Lenograstim for Febrile Neutropenia with Solid Tumors

blood product transfusion were used in 75 of the cases.

Cases that received and did not receive prophylactic G-CSF showed similar demographic characteristics (age, bone marrow involvement, first neutropenia development cycle, initial leukocyte, neutrophil, and platelet levels). However, while the rates of women and men were close to each other in the group receiving prophylaxis, majority of those that did not receive prophylaxis were women
(Table 1).Comparing the cases receiving and not receiving prophylaxis; it was observed that parameters followed during the febrile neutropenia treatment such as the need for a dose change in chemotherapy due to neutropenia, day of recovery from neutropenia, duration of hospitalization, and duration of using antibiotics were all similar ( $p>0.05)$. On the other hand, the number of days with fever was lower in the group that did not receive prophylaxis

Table 2. Features of Cases According to the G-CSF type being Given During the Febrile Neutropenia Attack

\begin{tabular}{|c|c|c|c|}
\hline & $\begin{array}{l}\text { Filgrastim group } \\
\qquad n=131\end{array}$ & $\begin{array}{l}\text { Lenograstim group } \\
n=44\end{array}$ & $\mathrm{p}$ value \\
\hline Number of cases $(\mathrm{M} / \mathrm{F})$ & $65 / 66$ & $21 / 23$ & 0.82 \\
\hline Age & $52.45 \pm 15.54$ & $53.13 \pm 17.05$ & 0.76 \\
\hline Bone marrow involvement (N/A-A) & $37 / 7$ (87 not examined) & $31 / 1$ (12 not examined) & 0.07 \\
\hline Initial chemotherapy dose (Exact/decreased) & $104 / 27$ & $36 / 8$ & 0.72 \\
\hline \multicolumn{4}{|l|}{ Neutropenia development cycle } \\
\hline $1-2^{\text {nd }}$ cycle & 87 & 23 & 0.09 (1-2 and other) \\
\hline $3-4^{\text {th }}$ cycle & 27 & 15 & 0.91 (1-4 and other) \\
\hline $5-6^{\text {th }}$ cycle & 13 & 4 & \\
\hline $7+$ & 4 & 2 & \\
\hline How many days after the chemotherapy (Mean \pm SD) & $10.19 \pm 3.79$ & $9.45 \pm 4.01$ & 0.31 \\
\hline Days with fever (Mean \pm SD) & $2.27 \pm 1.63$ & $2.73 \pm 2.40$ & 0.44 \\
\hline Comorbid disease (N/A-A) & $75 / 56$ & $23 / 21$ & 0.56 \\
\hline Leukocyte $(<1000 />1000)$ & $94 / 37$ & $36 / 8$ & 0.18 \\
\hline Neutrophil $(<500 />500)$ & $117 / 14$ & $43 / 1$ & $\begin{array}{c}0.11 \text { (Fischer exact, } \\
2 \text { tailed) }\end{array}$ \\
\hline Platelet $(\leq 50000 / \geq 50000)$ & $32 / 99$ & Oct-34 & 0.81 \\
\hline$(\leq 75000 / \geq 75000)$ & $41 / 90$ & $16 / 28$ & 0.53 \\
\hline Days of hospitalization (Mean) & $7.79 \pm 5.93$ & $7.75 \pm 4.63$ & 0.42 \\
\hline Day of recovery from neutropenia (Mean) & $3.31 \pm 1.55$ & $4 \pm 1.79$ & 0.02 \\
\hline Day of G-CSF received on hospitalization (Mean) & $4.31 \pm 2.02$ & $5.46 \pm 2.36$ & 0.002 \\
\hline Duration of antibiotics (Mean) & $7.36 \pm 5.10$ & $7.66 \pm 4.85$ & 0.45 \\
\hline
\end{tabular}

*(Abbreviations; F:Female M:Male A: Available N/A: Not Available)

Table 1. Demographic and Treatment Follow-up Parameters of Cases that Received and Did not Receive Prophylactic G-CSF

\begin{tabular}{|c|c|c|c|}
\hline & $\begin{array}{l}\text { Receiving prophylaxis } \\
\mathrm{n}=105\end{array}$ & $\begin{array}{l}\text { Not receiving prophylaxis } \\
\qquad \mathrm{n}=75\end{array}$ & $\mathrm{p}$ value \\
\hline Number of cases $(\mathrm{M} / \mathrm{F})$ & $59 / 46$ & $29 / 46$ & 0.02 \\
\hline Age & $52.82 \pm 15.84$ & $52.89 \pm 15.77$ & 0.37 \\
\hline Bone marrow involvement (N/A-A) & 43/5 (57 not examined) & $25 / 3$ (47 not examined) & $\begin{array}{l}1.0 \text { (Fischer exact } \\
\text { test, 2-tailed) }\end{array}$ \\
\hline Initial chemotherapy dose (Exact/decreased) & $82 / 23$ & $61 / 14$ & 0.59 \\
\hline \multicolumn{4}{|l|}{ Neutropenia development cycle } \\
\hline $1-2^{\text {nd }}$ cycle & 59 & 52 & 0.07 (1-2 and other) \\
\hline $3-4^{\text {th }}$ cycle & 28 & 17 & 0.07 (1-4 and other) \\
\hline $5-6^{\text {th }}$ cycle & 12 & 6 & \\
\hline $7+$ & 6 & & \\
\hline How many days after the chemotherapy $($ Mean \pm SD) & $10.19 \pm 3.79$ & $9.53 \pm 4.01$ & 0.37 \\
\hline Days with fever $($ Mean \pm SD) & $2.52 \pm 1.81$ & $2.15 \pm 1.88$ & 0.03 \\
\hline Comorbid disease (N/A-A) & $58 / 47$ & $42 / 33$ & 0.91 \\
\hline Leukocyte $(<1000 />1000)$ & $82 / 23$ & $50 / 25$ & 0.08 \\
\hline Neutrophil $(<500 />500)$ & $96 / 9$ & $68 / 7$ & 0.85 \\
\hline \multicolumn{4}{|l|}{ Platelet } \\
\hline$(\leq 50000 / \geq 50000)$ & $25 / 80$ & $17 / 58$ & 0.85 \\
\hline$(\leq 75000 / \geq 75000)$ & $35 / 70$ & $22 / 53$ & 0.56 \\
\hline Days of hospitalization (Mean) & $7.74 \pm 6.03$ & $7.75 \pm 4.85$ & 0.55 \\
\hline Day of recovery from neutropenia (Mean) & $3.49 \pm 1.69$ & $3.55 \pm 169$ & 0.88 \\
\hline Day of G-CSF received on hospitalization (Mean) & $4.71 \pm 2.21$ & $4.40 \pm 2.08$ & 0.27 \\
\hline Chemotherapy dose modification (N/A-A) & $42 / 63$ & $22 / 53$ & 0.14 \\
\hline Duration of antibiotics (Mean) & $7.70 \pm 5.34$ & $7.14 \pm 4.43$ & 0.50 \\
\hline Transfusion (N/A-A) & $56 / 49$ & $45 / 30$ & 0.37 \\
\hline Number of pathogen determined & 12 & 12 & 0.37 \\
\hline *(Abbreviations; F:Female M:Male A: Available N/A: Not & ailable) & & \\
\hline
\end{tabular}


$(\mathrm{p}=0.03)$. Table 1 illustrates demographic findings of cases that received and did not receive prophylaxis and their follow-up parameters during febrile neutropenia attacks.

G-CSF was started for 175 of patients, who started to be followed with the diagnosis of febrile neutropenia, along with the first dose of antibiotic treatment. While filgrastim was preferred for 131 of the cases, lenograstim was started for 44 cases. Comparing the filgrastim group (FG) and lenograstim group (LG), it was observed that both groups had similar demographic characteristics $(p>0.05)$. However, the day of recovery from neutropenia and the day of G-CSF given during the treatment were lower in the FG (Table 2).

Infection agent was determined in 24 of febrile neutropenia attacks. While G-CSF prophylaxis was given to $12(50 \%)$ of cases in which an infection agent was determined, others were not given the G-CSF prophylaxis. 21 of determined pathogens were bacteria-origin and 3 were fungi. All the fungi determined as pathogens were observed on patients receiving prophylactic G-CSF $(\mathrm{p}=0.21)$.

\section{Discussion}

The purpose of this study was to compare the filgrastim and lenograstim treatment being started during the FEN attack in patients with solid tumor that were retrospectively hospitalized and followed up with the diagnosis of FEN. FG had a faster recovery from neutropenia compared to LG (3.31 \pm 1.55 days $v s 4 \pm 1.79$ days; $\mathrm{p}=0.02)$ and received this treatment for a shorter time ( $4.31 \pm 2.02$ days vs. $5.46 \pm 2.36$ days; $p=0.002)$. While studies comparing both drugs generally involve treatments that are started for prophylaxis, this study compares the treatment that is given during the FEN attack. As a matter of fact, Kim et al. (2003) determined a faster recovery in the neutropenia in cases receiving a high dose of chemotherapy and having autologous peripheral blood stem cell transplantation, compared to filgrastim and lenograstim given as prophylactic (13.2 \pm 8 days $v s 19 \pm 10$ days; $p=0.004$ ) (Kim et al., 2003).

The neutrophils have an indisputable place in the natural immune response. Experimental studies have demonstrated that some defects occur in removing the bacteria from the focus of infection when the neutrophil count in the circulation decreases and the infection is successfully brought under control when the neutrophil count becomes normal again (Craig et al., 2009). A severe neutropenia duration also has a negative effect on the risk of infection (Procopio et al.,2011). Thus, the speed of recovery from neutropenia in neutropenic patients will increase the speed of controlling the infection. In this study, it was observed that neutropenia had a faster recovery in patients receiving filgrastim compared to those receiving lenograstim. Thus, considering a G-CSF supplement to the treatment in old patients with a weak performance or nutrition, comorbid disease and a severe infection, we thought that filgrastim is preferred.

It is one of the important parameters being taken into consideration in the treatment stage of cost-effective diseases. In Turkey, the daily treatment cost of lenograstim is higher compared to filgrastim (Sari et al., 2013). In their study comparing both drugs in terms of cost-efficacy, Gardellini A et al. also determined that lenograstim had a higher cost than filgrastim (Gardellini et al., 2013). Within this study, no cost analysis was conducted. However, considering that filgrastim has a fewer usage days than lenograstim, hospitalization durations are similar in both groups and price of lenograstim is higher than filgrastim, it could be asserted that the choice of lenograstim would cause a greater cost than the choice of filgrastim.

Febrile neutropenia is generally encountered in first cycles of the myelosuppressive treatment (Procopio et al., 2011). It was observed after the first two cycles in $61.7 \%$ of FEN attacks of our cases. The FEN attack was observed averagely after 10.19 days in patients receiving prophylactic G-CSF and after 9.53 days in patients receiving none. However, this difference was not statistically significant. The both groups also had a similarity in terms of the day of recovery from neutropenia, day of G-CSF received during the FEN attack, duration of antibiotics used and hospitalization duration ( $>0.05$ ). However, the number of days with fever was greater in those who had previously received either primary or secondary G-CSF prophylaxis ( $\mathrm{p}=0.03$ ). Since patients receiving primary or secondary prophylaxis are inclined to FEN due to either chemotherapy (group receiving a high-risk ct) or their performance states, we think that the excess number of days with fever is an expected state in this fragile patient group. Our study has some limitations. It is primarily observed that the groups receiving filgrastim and lenograstim did not have a balanced distribution; majority of patients were involved in the filgrastim group. This condition is caused by the retrospective design of the study. Besides, this study could not determine the cases that did not develop the FEN attack independently from using prophylactic G-CSF or not. If we determined all of those cases, we could reveal the effect of prophylactic G-CSF on the frequency and course of FEN more precisely. Similarly, since it is not possible to know the type of G-CSF given in prophylaxis; information about whether the type of G-CSF affects the course of FEN or not could not be reached.

As a consequence, filgrastim is more effective on recovering the neutropenia during the FEN attack compared to lenograstim; and a shorter period and lower treatment cost are required in recovering the neutropenia.

\section{References}

Aapro MS, Bohlius J, Cameron DA, et al (2011). European organization for research and treatment of cancer. 2010 update of EORTC guidelines for the use of granulocytecolony stimulating factor to reduce the incidence of chemotherapy-induced febrile neutropenia in adult patients with lymphoproliferative disorders and solid tumors. Eur $J$ Cancer, 47, 8-32.

Clark OA, Lyman GH, Castro AA, et al (2005). Colonystimulating factors for chemotherapy-induced febrile neutropenia: a meta-analysis of randomized controlled trials. J Clin Oncol, 23, 4198-214.

Cooper KL, Madan J, Whyte S, et al (2011). Granulocyte colonystimulating factors for febrile neutropenia prophylaxis 
following chemotherapy: systematic review and metaanalysis. BMC Cancer, 11, 404

Craig A, Mai J, Cai S, et al (2009). Neutrophil recruitment to the lungs during bacterial pneumonia. Infect Immun, 77, 568-75.

Freifeld AG, Bow EJ, Sepkowitz KA, et al (2011). Infectious diseases society of america. clinical practice guideline for the use of antimicrobial agents in neutropenic patients with cancer: 2010 update by the infectious diseases society of America. Clin Infect Dis, 52, 56-93.

Fontanella C, Bolzonello S, Lederer B, Aprile G (2014). Management of breast cancer patients with chemotherapyinduced neutropenia or febrileneutropenia. Breast Care (Basel), 9, 239-45

Gardellini A, Gigli F, Babic A, et al (2013). Filgrastim XM02 $\left(\right.$ Tevagrastim ${ }^{\circledR}$ ) after autologous stem cell transplantation compared to lenograstim: favorable cost-efficacy analysis. Ecancermedicalscience, 7, 327.

Hughes WT, Armstrong D, Bodey GP, et al (2002). Guidelines for the use of antimicrobial agents in neutropenic patients with cancer. Clin Infect Dis, 34, 730-51.

Kim IH, Park SK, Suh OK, et al (2003). Comparison of lenogastrim and filgastrim on haematological effects after autologous peripheral blood stem cell transplantation with high-dose chemotherapy. Current Med Res Opinion, 19, 753-59

Orciuolo E, Buda G, Marturano E, et al (2011). Lenograstim reduces the incidence of febrile episodes, when compared with filgrastim, in multiple myeloma patients undergoing stem cell mobilization. Leuk Res, 35, 899-903.

Osmani AH, Ansari TZ, Masood N, et al (2012). Outcome of febrile neutropenic patients on granulocyte colony stimulating factor in a tertiary care hospital. Asian Pac J Cancer Prev, 13, 2523-26.

Procopio G, Niger M, Testa I (2011). Lecture: management of chemotherapy-induced febrile neutropenia; guidelines and colony stimulating factors. Neurol Sci, 2, 217-19.

Sari N, Dalva K, Ilhan IE (2013). Comparison of filgrastim and lenograstim in pediatric solid tumors. Pediatr Hematol Oncol, 30, 655-61

Serkan Keskin E (2012). The use of high-dose chemotherapy and hematopoietic growth factors. In Mandel NM, Onat $\mathrm{H}(\mathrm{ed})$ : Cancer Patient Approach. ( $2^{\text {nd }}$ ed) Istanbul, Nobel Tip Kitabevi, 15, 155-61 (in Turkish).. 